\title{
Size-Selected Clusters as Model Systems for Catalysis
}

\author{
Knut R. Asmis ${ }^{1} \cdot$ André Fielicke $^{2}$
}

Published online: 7 March 2018

(c) Springer Science+Business Media, LLC, part of Springer Nature 2018

The aim of this special issue is to highlight current experimental techniques that exploit size-selected clusters, predominantly in the gas phase, as model systems for understanding molecular-level processes relevant in heterogeneous catalysis. The metal and metal oxide clusters of relevance here, in principle, are relatively simple systems containing often only a few metal atoms (or sometimes even only a single) and are well defined in terms of their composition, size and charge state. Such cluster studies are always motivated by a fundamental curiosity for the properties of this intermediate form of matter. However, it is the transfer of molecularlevel insights into the structure, stability and reactivity of isolated, stabilized or supported clusters to unravel more complex problems related to the local properties of reaction sites encountered in surface chemistry and ultimately catalysis, that makes these studies potentially interdisciplinary and transcending.

Such ideas are, of course, not really new, but have been emphasized already, for instance, by Schumacher et al. and Muetterties et al. in the 1970s for free and ligand-stabilized metal clusters, respectively $[1,2]$. As a result of the pioneering work of the groups of Smalley, Cox and Kaldor, Riley, Wöste and others the interest in clusters and specifically their chemical behavior increased significantly in the 1980s/1990s due to new possibilities for synthesizing gasphase metal clusters in a wide size range, depositing them on metal oxide supports, characterizing them by different ways of spectroscopy and studying their size-dependent reactivities [3-5]. With the beginning of the twenty-first century, increasingly more sophisticated experimental (as well as

André Fielicke

fielicke@fhi-berlin.mpg.de

Knut R. Asmis

knut.asmis@uni-leipzig.de

1 Wilhelm-Oswald Institut für Physikalische und Theoretische Chemie, Universität Leipzig, Linnéstr. 2, 04103 Leipzig, Germany

2 Fritz-Haber-Institut der Max-Planck-Gesellschaft, Faradayweg 4-6, 14195 Berlin, Germany theoretical) techniques have been applied to the study of reactions involving size-selected clusters. These have delivered profound insights into reaction mechanisms and have unraveled complex reaction cycles catalyzed by metal-containing clusters [6-9].

Experiments on gas-phase clusters are typically not intended to mimic reactions occurring in real catalysts under process conditions. The substantially reduced complexity in such model systems, however, allows isolating specific aspects of a reaction mechanism at the molecular level. Moreover, a particular attractive aspect of small- and medium-sized clusters is that their properties are amenable to high level quantum chemical methods. Therefore, a central aspect of this type of cluster research is to test, benchmark and evaluate modern electronic structure methods for their potential use in describing processes in more complex systems. The essential role of theory for interpreting and thoroughly understanding experimental results becomes quite obvious in many of the contributions published here.

This issue consists of a (small) collection of original research articles, which hopefully represent a cross section of some of the most current experimental methods applied to the general topic sketched above. The investigated systems span the ranges of size and complexity, from the seemingly simplest case, complexes of single metal atoms, over isolated pure and binary metal as well as metal oxide clusters, towards stabilized and immobilized nanoclusters. The contributions highlight, how the chemistry of clusters is probed experimentally, e.g. how reaction kinetics and energetics are quantified and the geometric as well as electronic structure of the reaction participants are characterized exploiting the vast arsenal of highly sensitive and selective mass spectrometric and spectroscopic techniques.

Summarizing, such a collection can never replace a comprehensive review of the field, and this is not intended, but by collecting snapshots of current research work performed by experts in the field it hopefully gives an alternative overview of the state-of-the-art of the field and thereby, as a whole, complements more classical reviews. 
We are grateful to all authors that they share their latest results with us to be published in Topics in Catalysis and thank the Editors-in-Chief for initiating this topical issue.

\section{References}

1. Muetterties EL, Rhodin TN, Band E, Brucker CF, Pretzer WR (1979) Clusters and surfaces. Chem Rev 79:91

2. Schumacher E, Kappes M, Marti K, Radi P, Schaer M, Schmidhalter B (1984) Metal clusters: preparation, properties, theory. Ber Bunsenges Phys Chem 88:220

3. Knickelbein MB (1999) Reactions of transition metal clusters with small molecules. Ann Rev Phys Chem 50:79
4. Duncan MA (2000) Frontiers in the spectroscopy of mass-selected molecular ions. Int J Mass Spectrom 200:545

5. Meiwes-Broer KH (ed) (2000) Metal clusters at surfaces: structure, quantum properties, physical chemistry. Springer, Berlin

6. Böhme DK, Schwarz H (2005) Gas-phase catalysis by atomic and cluster metal ions: the ultimate single-site catalysts. Angew Chem Int Ed 44:2336

7. Woodruff DP (ed) (2007) Atomic clusters: from gas phase to deposited. Elsevier, Amsterdam

8. Heiz U, Landman U (eds) (2007) Nanocatalysis. Springer, Berlin

9. Luo Z, Castleman AW, Khanna SN (2016) Reactivity of metal clusters. Chem Rev 116:14456 